\title{
TRATAMIENTO QUIRÚRGICO DEL COMPLEJO EXTROFIA-EPISPADIAS. REVISIÓN Y CONCEPTOS ACTUALES
}

\author{
E. DE LA PEÑA, J. HIDALGO, J. CAFFARATTI, J.M.GARAT, H. VILLAVICENCIO \\ Unidad de Urología Pediátrica. Servicio de Urología. Fundació Puigvert. Barcelona.
}

Actas Urol Esp. 27 (6): 450-457, 2003

\section{RESUMEN \\ TRATAMIENTO QUIRÚRGICO DEL COMPLEJO EXTROFIA-EPISPADIAS. REVISIÓN Y CONCEPTOS ACTUALES}

Desde sus descripciones iniciales el tratamiento quirúrgico del complejo extrofia-epispadias (CEE) ha sufrido paulatinas modificaciones en busca de unos buenos resultados estéticos y funcionales. Los primeros procedimientos de derivación urinaria y exéresis de la placa vesical fueron evolucionando de la mano de diferentes autores mejorando o complementando los avances anteriormente descritos, llegando hasta el cierre vesical por etapas y más recientemente el cierre completo primario del CEE. Estas últimas técnicas permiten obtener buenos índices de continencia y satisfactorios resultados estéticos; sin embargo la baja incidencia y la complejidad de la patología, tanto en su tratamiento inicial, como en sus complicaciones, aconsejan manejarla en centros de referencia.

Participando de este espíritu de combinación de técnicas, en busca de un resultado satisfactorio para estos recién nacidos extróficos e inspirados en la contribución de Leadbetter al procedimiento de YoungDees para la corrección del reflujo vesicoureteral (RVU), hemos añadido un nuevo paso al cierre completo primario del CEE conocido como técnica de Mitchell, realizando una trigonoplastia de Gil-Vernet, para la prevención del RVU, íntimamente asociado a la extrofia vesical.

Consideramos que el RVU puede prevenirse en el primer manejo quirúrgico del recién nacido extrófico.

PALABRAS CLAVE: Extrofia vesical. Reflujo vesicoureteral. Prevención.

\section{ABSTRACT}

SURGICAL TREATMENT OF EXSTROPHY-EPISPADIAS COMPLEX. REVIEW AND NEW CONCEPTS

Since earlier descriptions the surgical management of the bladder exstrophy and epispadias complex (BEEC) has experimented gradual changes in the search for good aesthetic and functional results. The initial urinary diversion and bladder plate removal has evolved under various authors either by improving or supplementing the above described advances, until reaching the staged functional closure and more recently the complete primary BEEC closure. These later techniques allow to obtain encouraging continence indexes and successful aesthetic results; the low incidence and complexity of this condition however, advises both in terms of early management and later complications that this should be managed in reference centres.

Sharing on this spirit of technique combination in search for a satisfactory result for exstrophic newborns and inspired by Leadbetter's contribution to Young-Dees's procedure for vesicoureteral reflux (VUR) correction, we have added a new step to the complete primary BEEC closure known as the Mitchell's technique by performing Gil-Vernet's trigonoplasty to prevent VUR, closely associated to bladder exstrophy.

We believe VUR can be prevented right from the first surgical approach in the exstrophic newborn.

KEY WORDS: Bladder exstrophy. Vesicoureteral reflux. Prevention. 
$\mathrm{E}$ tratamiento del recién nacido con extrofia vesical continua siendo uno de los retos más difíciles para el urólogo.

Esta peculiar patología se presenta en uno de cada 10.000 a 50.000 recién nacidos, con una mayor frecuencia en niños ${ }^{1}$. El tratamiento del CEE es todavía objeto de controversia.

La reconstrucción por etapas basada en la cistorrafia y abdominoplastia, la reconstrucción del cuello vesical y la posterior uretroplastia y/o clitoroplastia ha dado paso al manejo quirúrgico actual preconizado por Mitchell ${ }^{1}$ basado en la combinación de estas diferentes etapas en una única reconstrucción anatómica cuyos objetivos son un cierre vesical inicial seguro, la reconstrucción de unos genitales externos aceptables cosmética y funcionalmente y sobre todo la mejoría de los índices de continencia urinaria con la preservación al mismo tiempo de una adecuada función renal ${ }^{2}$. Mitchell combina estas secuencias en una sola intervención con la movilización de la vejiga y la uretra posteriormente hasta situarlas en una posición anatómica normal.

El reflujo vesicoureteral es una patología intimamente asociada a la extrofia vesical. Dada la elevada incidencia, la mayoría de los autores prescriben antibióticos en dosis bajas a todos los recién nacidos después de la intervención. Incluso Mitchell defiende su corrección quirúrgica mediante un reimplante dado el alto índice de episodios de pielonefritis descritos en su serie $^{1}$.
En este trabajo realizamos una revisión sobre las diferentes formas de tratamiento quirúrgico, desde sus primeras descripciones hasta el actual manejo, completándolo con nuestra propia aportación a la técnica descrita por Mitchell ${ }^{3}$.

\section{MANEJ O PERINATAL}

La extrofia vesical, la extrofia cloacal y el epispadias son variantes del complejo extrofiaepispadias. Este gran espectro de anomalias es la representación de distintos grados de un mismo defecto embriológico, al que han sido atribuidas diferentes teorías sobre su desarrollo malformativo.

Cabe la pena destacar una serie de consideraciones sobre el manejo de la placa extrófica en el momento postnatal inmediato. Tras el nacimiento, la mucosa vesical suele ser lisa, delgada e intacta, aunque hay que recordar que es muy sensible y se erosiona con facilidad. Por eso, es recomendable seguir dos consejos:

1. El cordón umbilical debe ser ligado con suturas de nylon o de material reabsorbible, lo más cerca posible de la pared abdominal, evitando el uso de clips umbilicales, que pueden traumatizar la mucosa. (Fig. 1).

2. Además la placa vesical debe ser cubierta de un material no adhesivo e hidrofílico, evitando las gasas o apósitos vaselinados que pueden secarse y producir avulsiones de la mucosa cuando son retirados.
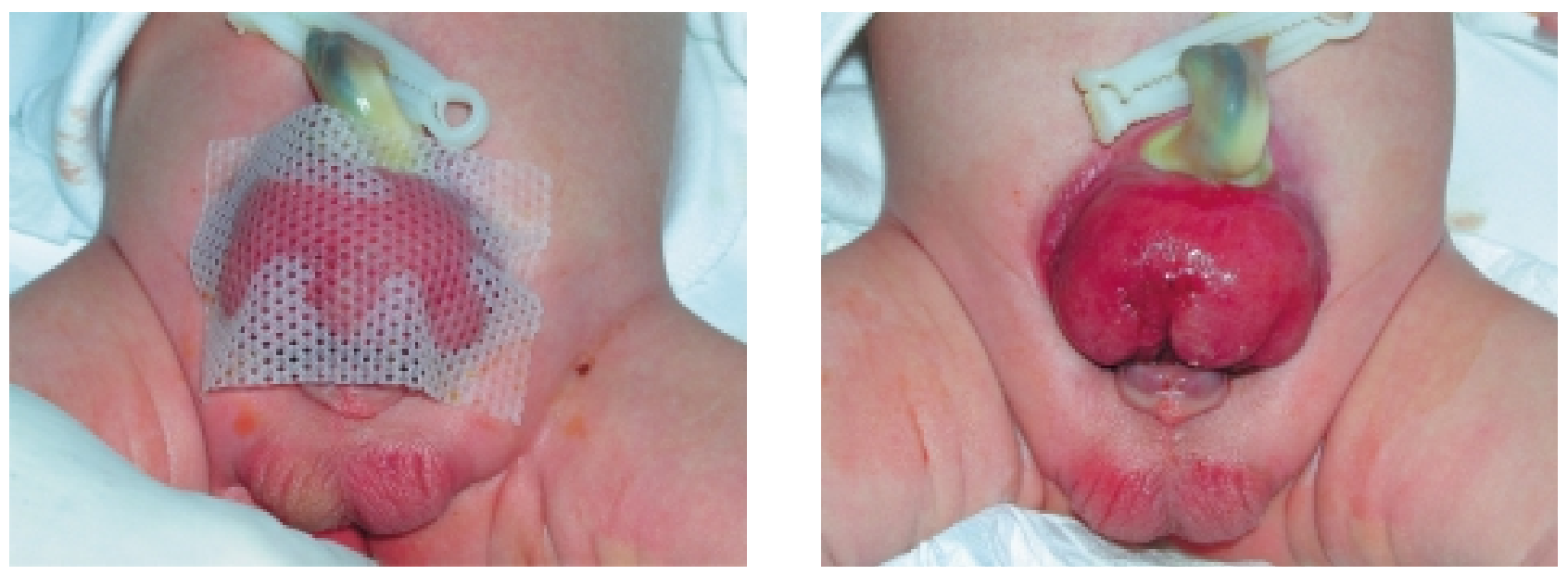

FIGURA 1: No resulta recomendable el uso de pinzas de plástico para la ligadura del cordón umbilical y si una cobertura de la placa con material siliconado que evite la escoriación de la mucosa vesical (tipo Mepitel ${ }^{\circledR}$ ). 
Aunque el diagnóstico prenatal es sensible y específico, se precisa de un correcto entrenamiento y una adecuada paciencia del examinador para completar un estudio ultrasonográfico en el que se pueda visualizar la vejiga fetal en sus fases de llenado y vaciado. De esta manera, el personal de la sala de partos podrá estar preparado para el correcto tratamiento del neonato e incluso organizado el traslado a un centro de referencia en el manejo de la extrofia vesical.

\section{NUEVOS CONCEPTOS EN EL MANEJ O QUIRÚRGICO DE LA EXTROFIA VESICAL}

Al margen de las primeras descripciones quirúrgicas realizadas a finales del siglo XIX por Trendelenburg ${ }^{1}$, entre otros, la verdad es que durante la primera mitad del siglo pasado, los pacientes eran sencillamente clasificados en dos grupos: aquellos en los que se intentaban el cierre primario por la bondad y la distensibilidad de la placa y aquellos otros en los que directamente se decidía una derivación urinaria. A este respecto, se han descrito innumerables tipos de técnicas: ureterosigmoidostomías, conductos ileales, conductos colónicos ${ }^{4-7}$.

Young ${ }^{8}$, es el primero en preconizar el cierre vesical primario con corrección además del epispadias de la mayor parte de los recién nacidos; sin embargo, los resultados eran mediocres, fundamentalmente por fallos de la técnica quirúrgica, con tasas de continencia muy bajas y altos indices de insuficiencia renal.

Más tarde, Jeffs $^{9}$ movido por un cambio de actitud en el manejo del niño extrófico, aboga por el cierre primario de todos los recién nacidos con extrofia vesical, con el objetivo de realizar un cierre seguro de la pared abdominal, alcanzar una continencia urinaria con una correcta preservación de la función renal y por último la reconstrucción, en el paciente varón, de un pene aceptable desde un punto de vista funcional y estético. Así se describe la reparación en etapas, realizando el cierre vesical con aproximación de la sínfisis púbica a la que se añade, si es necesario, la osteotomía en el periodo neonatal, lo que facilita un vaciado de la vejiga a baja presión y como consecuencia, una protección del tramo urinario superior. Posteriormente, se realiza la recons- trucción del cuello vesical a los 4 años, y se completa el cierre por etapas con la corrección del epispadias.

Tras los primeros procedimientos realizados, Jeffs observó el hecho de que la vejiga se vaciaba sin ninguna resistencia, produciendo vejigas desfuncionalizadas y de baja capacidad que comprometían la posterior reconstrucción del cuello vesical. Por todo lo expuesto Jeffs realiza un cambio en el orden de este cierre por etapas, anteponiendo la corrección del epispadias a la reconstrucción del cuello vesical; de esta manera se conseguía añadir cierto grado de resistencia al vaciado vesical y con ello aumentar la capacidad de la vejiga para realizar con garantías una reconstrucción del cuello vesical según la técnica de Young-Dees a la que se asocia además un reimplante ureteral tipo Cohen ${ }^{10}$ dado el alto índice de reflujos vesicoureterales en estos pacientes. La decisión final de hacer una trigonocervicouretroplastia es comprometida y en numerosas ocasiones, se precisa de la realización de una ampliación vesical, decisión que en última instancia se toma en el acto operatorio, aunque es controvertido si su realización se debe realizar previa o posteriormente a la reconstrucción del cuello vesical. Se aplican algunos principios clínicos para la toma de esta decisión, como son una capacidad vesical de al menos 60ml, la ausencia de hidronefrosis y lesiones parenquimatosas secundarias a pielonefritis y que se tenga una aproximación adecuada de la sínfisis pubiana. Todo ello está enmarcado en el cuadro de un estudio urodinámico difícil de aplicar y más difícil de interpretar adecuadamente.

Para la realización de la cervicouretroplastia, es necesario utilizar parte de la superficie vesical, lo que compromete posteriormente un cierre con un adecuado volumen vesical que no afecte el tramo urinario superior por elevadas presiones. El mecanismo de continencia esta basado el principio de Lapides ${ }^{11}$, basado en la ley de Laplace (la resistencia al paso de un líquido por un tubo está en relación directa con la longitud del mismo y con la tensión de sus paredes y en relación inversa del diámetro de su luz)

Además de esto, la cervicouretroplastia se completa con una técnica de anclaje desde la 
cara superior de la uretra y cuello vesical, a la superficie posterior de la sínfisis pubiana, según la técnica de Marshall-Marchetti-Krantz ${ }^{12}$

Martínez Piñeiro ${ }^{13}$ en 1962 describe la utilización de un segmento sigmoideo no destubularizado anastomosado a la neouretra tubulizada mediante cervicotrigonouretroplastia en una niña, y también describe la utilización de sigmoides destubularizado, aplicando los principios quirúrgicos de Young en el varón ${ }^{14}$. Resulta fundamental la aportación de Leadbetter ${ }^{15}$ al procedimiento descrito por Young y Dees al realizar la reimplantación ureteral mediante un túnel submucoso superiormente, lo que permite la utilización completa del trígono vesical para el remodelaje cervical.

Arap ${ }^{16}$ realiza también aportaciones importantes en la descripción de una nueva técnica por etapas basada en la creación de un conducto colónico con reimplantación ureteral antireflujo que posteriormente es usado como colocistoplastia no destubularizada. Este hecho compromete la continencia final de esta técnica. El sigmoides se anastomosaría a una neouretra de 5 ó $6 \mathrm{~cm}$ creada a partir de la placa vesical. La pared abdominal es reconstruida mediante colgajos de tejidos paraextróficos según describió en su técnica Allen ${ }^{17}$.

Otros autores como Jones ${ }^{18}$ o Mollard ${ }^{19}$ propusieron variantes de esta cervicouretroplastia, diferentes a la descritas pero que en definitiva también implicaban una reducción considerable de la capacidad vesical.

Mediante esta maniobra y según los autores que se revisen, los índices de continencia urinaria podrían variar del 10 al $88 \%$ con una tasa relativamente baja de fracaso renal entorno al 13 o $20 \%{ }^{20}$. Se han descrito unos resultados favorables con la técnica de Young-Dees del $70 \%^{2}$, entendidos estos buenos resultados como una aceptable continencia urinaria con preservación de la función renal. Con esta técnica pueden aparecer un $20 \%$ de complicaciones mayores, como son: el prolapso vesical, la dehiscencia del cierre, o la hidronefrosis que obligan en la mayoría de los casos a repetir el procedimiento quirúrgico. Se han descrito un $10 \%$ de complicaciones menores como son: la litiasis, la infección y supuración de la sutura pubiana, fístulas urinarias y la metaplasia escamosa del reborde superior del cierre epispádico. Estas complicaciones de tipo menor pueden ser razonablemente resueltas sin comprometer el cierre vesical ni la trigonocervicouretroplastia ${ }^{21-23}$.

\section{EL PAPEL DE LA OSTEOTOMÍA}

Todos los casos de extrofia vesical presentan una diástasis característica de la sínfisis púbica causada por la rotación externa de los huesos innominados sobre las articulaciones sacroilíacas en relación con el plano sagital del cuerpo. Además, se produce la rotación externa o eversión de las ramas del pubis en su unión con los huesos isquiático e ilíaco. Para algunos autores, el cierre vesical seguro exigía la realización de una osteotomía ilíaca posterior que permitiese una rotación interna de las palas ilíacas en la aproximación a la línea media de la sínfisis púbica.

Hoy en día, esta maniobra y sus variantes, continúan siendo controvertidas, habiéndose restringido su uso en pacientes mayores de un mes, que presentan gran diástasis púbica $\mathrm{y}$, sobre todo, si ha fracasado un primer cierre vesical ${ }^{21,22}$.

Estos mismos autores, consideran que la osteotomía mejora luego los resultados de la continencia y el aspecto final del tallo peneano al aumentar su longitud. Sin embargo, continúa abierto el debate en cuanto al tiempo de realización de esta técnica, discutiéndose si hacerlo simultáneamente al cierre quirúrgico o dos o tres semanas después del cierre.

Desde el punto de vista quirúrgico, merece la pena destacar que el $80 \%$ de los niños y el 10$15 \%$ de las niñas presentan hernia inguinal al nacimiento o posteriormente a la reconstrucción del complejo extrofia-epispadias, por lo que algunos autores proponen cerrar el conducto herniario en el mismo momento del cierre vesical, mediante un abordaje preperitoneal ${ }^{24,25}$ Entre un $15 \%$ a un $50 \%$ según las series, necesitan posteriormente una herniorrafia con carácter de urgencia por incarceración ${ }^{26,27}$.

\section{EL TRATAMIENTO DEL EPISPADIAS}

En cuanto al tratamiento del epispadias, las aportaciones más destacadas las realiza Ransley ${ }^{28}$ a partir de la primitiva técnica de Cantwell ${ }^{29}$. En 


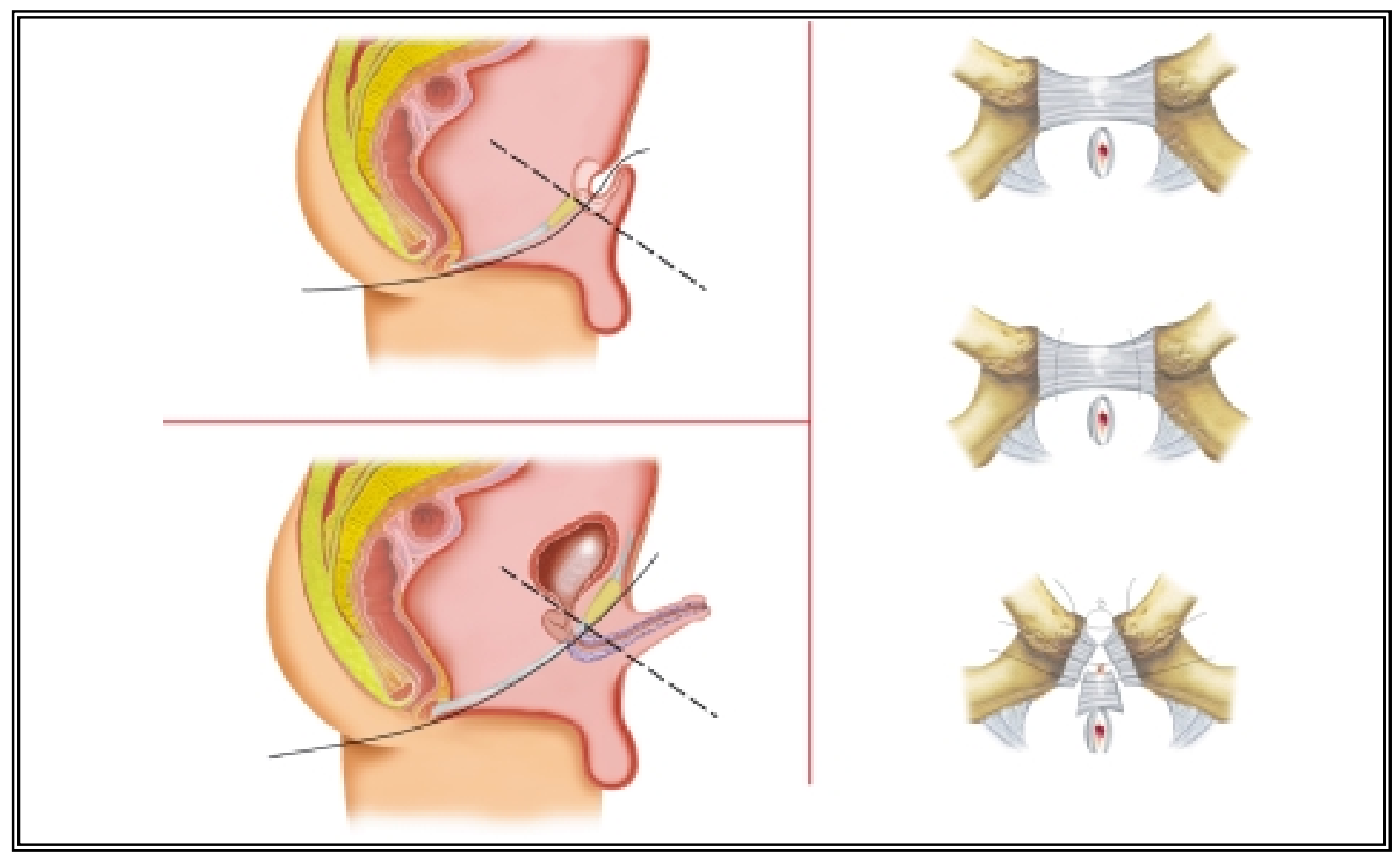

DIBUJO 1 : (a,b y c) La resección del rudimento intersinfisario permite la aproximación a la linea media de la sinfisis púbica, por debajo de la cual queda dispuesto el cierre vesical1,2. De esta manera, el cuello vesical y la uretra son desplazados posteriormente hasta una posición anatómica normal.

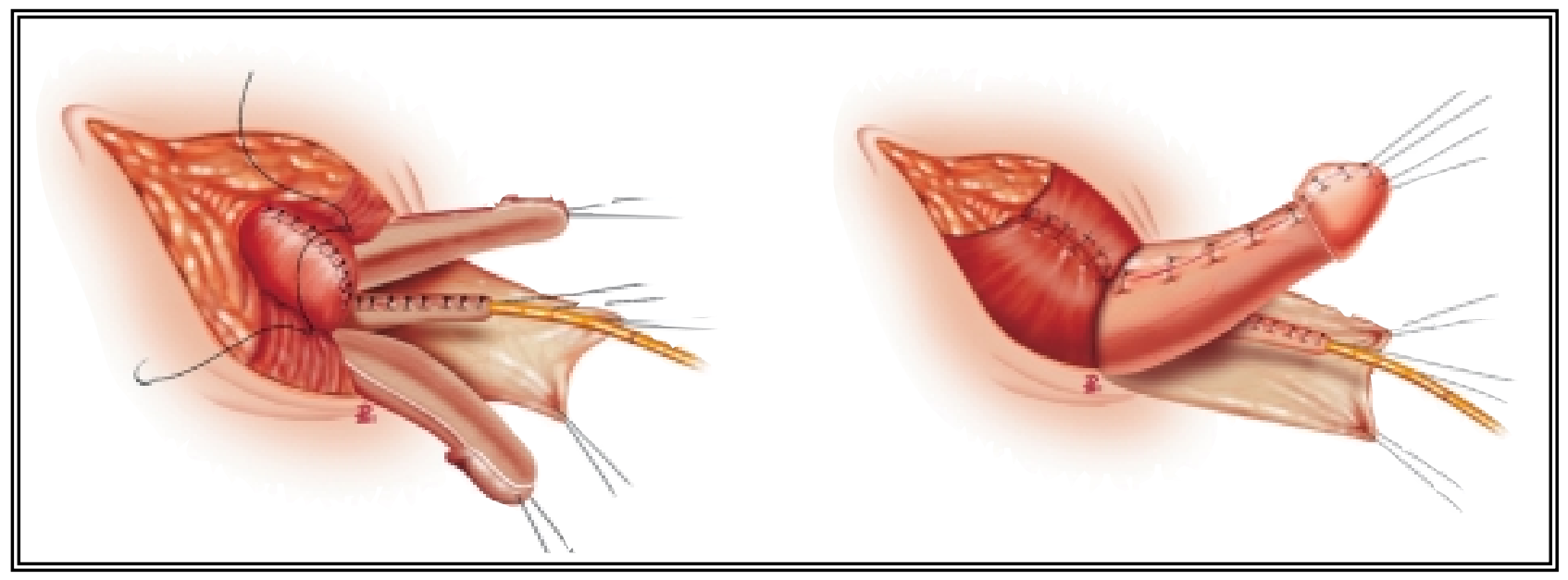

DIBUJO 2: (a). El cierre vesical y el uretral se realizan de forma continua tras separar la placa uretral de los cuerpos cavernosos y estos entre si, de tal manera que las tres estructuras quedan independizadas. Esto permite ventralizar la neouretra tubularizada. (b). Tras desrotar los cuerpos cavernosos y suturarlos en la linea media el cuello vesical y la uretra se desplazan posteriormente y sobre ellos se aproxima el rudimento sinfisario.

1985 describe una técnica quirúrgica basada en los principios de preservación de la placa uretral, ventralización de la neouretra y rotación de los cuerpos cavernosos; todo ello con la finalidad de mejorar el aspecto externo del pene a expensas de aumentar el tallo peneano y conseguir una normofunción miccional con la colocación de la uretra en el plano ventral peneano, es decir, reconstruir un pene lo más parecido a lo normal, preservando la placa uretral. 


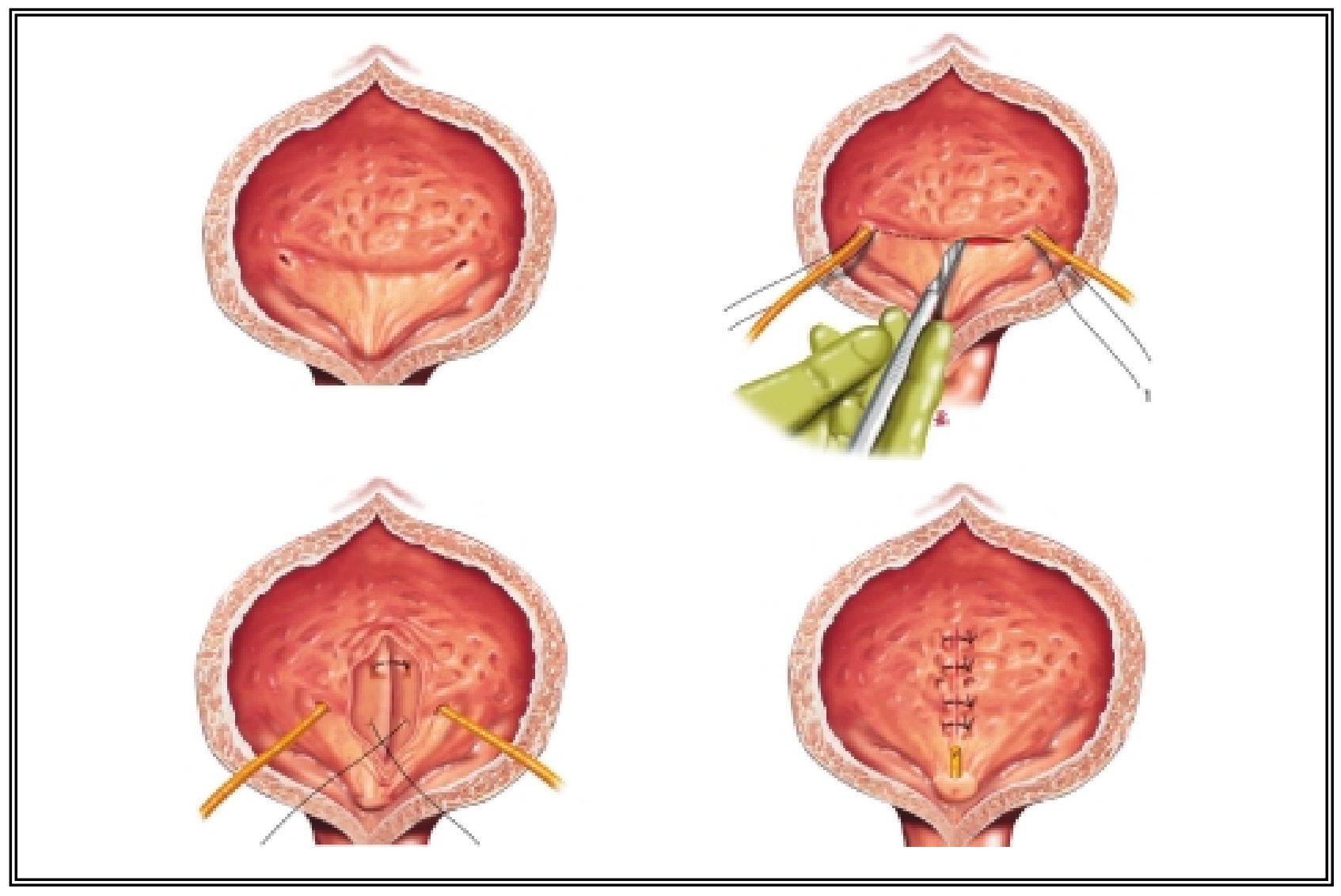

DIBUJO 3: Trigonoplastia según técnica de Gil-Vernet.

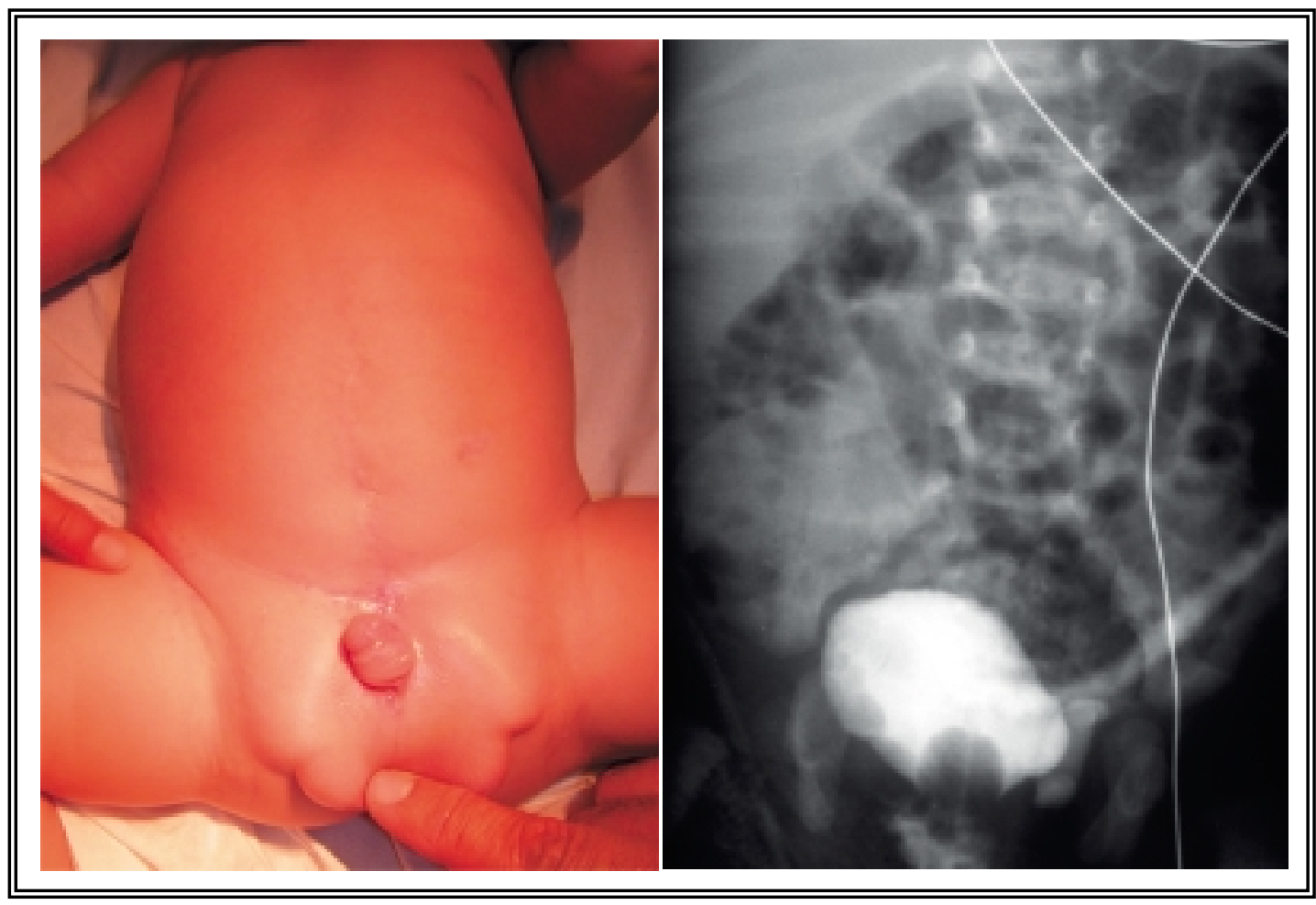

FIGURA 2: Resultado estético final a los tres meses de la cirugia del cierre del CEE del mismo paciente de la imagen 1, mediante un cierre primario completo. Cistografia de control que demuestra ausencia de RVU tras la trigonoplastia en este mismo paciente. 
Los pasos fundamentales de su técnica son: una corrección del meato distal con una maniobra similar a la realizada en la técnica de MAGPI para el hipospadias, una corrección del chordee dorsal mediante la disección de la placa uretral y su reconstrucción tubularizada, seguido de la desrotación de los cuerpos cavernosos y su aproximación a la línea media, mediante la realización de una anastomosis caverno-cavernosa. La cirugía se completa con la reconstrucción del glande y el cierre de la piel peneana mediante la utilización de un colgajo prepucial ventral que tras ser rotado, cubre la superficie dorsal peneana.

La tasa de éxitos es elevada presentando sólo un $8 \%$ de fístulas uretrocutáneas ${ }^{30}$, frente al 38\% de las descritas con la técnica de Young ${ }^{31}$ o el $30 \%$ de las de $\mathrm{Kramer}^{32}$.

\section{CORRECCIÓN SIMULTÁNEA DE LA EXTROFIA Y EPISPADIAS EN EL RECIÉN NACIDO}

No fue hasta hace unos años cuando se comunican los primeros resultados de una nueva técnica quirúrgica basada en la corrección simultánea de la extrofia vesical y el epispadias. Fueron Mitchell ${ }^{3}$ y McLorie $^{33}$ los que describen la combinación de las diferentes etapas descritas previamente por $\mathrm{Jeffs}^{9}$ en un solo procedimiento quirúrgico. Desde el punto de vista anatómico, esta técnica quirúrgica, está basada en dos puntos fundamentales: el primero de ellos presupone la transposición del cuello vesical y la uretra a un plano anatómico pélvico posterior más normal, tras haber realizado el cierre continuo de la vejiga, cuello vesical y uretra (Dibujo 1) Esta posición pelviana posterior favorece la aparición de un mecanismo de resistencia al vaciado que propicia la formación de unos ciclos de llenado y vaciado vesical, ciclos mecánicos que generan fuerzas de estiramiento del detrusor que potencian la producción de factores de crecimiento muscular. Todo ello favorece la aparición de una continencia urinaria precoz. A diferencia de la técnica por etapas de Jeffs, esta continencia se iría adquiriendo a lo largo del crecimiento del niño y no estaría supeditada a la realización de la cervicouretroplastia a los cuatro años, reforzando el entrenamiento miccional por la existencia de un fenómeno anatómico de continencia. Esto se lograría "profundizando" la uretra en la pelvis mediante la recolocación inicial del complejo cérvicouretral por detrás y por debajo de un plano resistente intersinfisario.

El segundo paso fundamental, desde el punto de vista anatómico de la técnica de Mitchell, es conocido como el "desmontaje" peneano. Según muestra el Dibujo 2, es necesario realizar la separación de los dos cuerpos cavernosos por la línea media incidiendo sobre el tejido fibroso avascular que los une. De esta manera, la placa uretral puede ser movilizada hacia el plano peneano ventral, los cuerpos cavernosos son desrotados, la longitud del tallo peneano aumenta y la corrección del glande es más anatómica.

Los resultados de esta técnica quirúrgica, han sido comunicados por Mitchell sobre una serie de 16 pacientes adquiriendo unos buenos índices de continencia urinaria cercanos al $88 \%$ y un ahorro considerable en el número de cirugías practicadas sobre estos pacientes ${ }^{2}$.

De la revisión de los resultados comunicados por Mitchell, llama la atención el alto índice de reintervenciones (12 de los 16 pacientes operados de su serie), por pielonefritis secundaria a reflujo vesico-ureteral ${ }^{1}$. Esto en principio, iría en contra de la filosofía promulgada por el autor, basada en la reducción del número de cirugías, realizadas sobre estos pacientes.

Por todo lo expuesto, nosotros decidimos incorporar un nuevo paso a la técnica descrita por Mitchell que supusiera una baja morbilidad quirúrgica con una tasa de resultados exitosos suficientemente avalada. Esta combinación supone una novedad no descrita previamente en la literatura revisada. La trigonoplastia, según la técnica de Gil-Vernet ${ }^{34}$ (Dibujo 3), ha obtenido buenos resultados en el $80 \%$ de los casos en las que ha sido aplicada ${ }^{35}$, lo que nos hace suponer que podría utilizarse de manera rutinaria como primer paso en el procedimiento de Mitchell para prevenir el reflujo. Es muy sencilla de realizar, no prolongando excesivamente el acto quirúrgico $\mathrm{y}$ con un indice de complicaciones añadidas muy bajo. Además esta técnica preserva la localización ipsilateral de cada meato, lo que facilita el abordaje en posteriores procedimientos endoscópicos, si son necesarios. 


\section{CONCLUSIONES}

El CEE supone un reto quirúrgico para el urólogo. Dada su baja incidencia y alta complejidad el recién nacido con extrofia debería ser tratado en centros de referencia con experiencia en su manejo.

A lo largo de la historia se han desarrollado diferentes técnicas quirúrgicas, cuyo último fin es el de proporcionar un adecuado resultado estético con satisfactorios índices de continencia, preservando la función renal.

En los casos en los que es posible realizar el cierre completo primario estos principios pueden ser alcanzados con una única intervención.

Creemos que es posible asociar una técnica de corrección del RVU en el mismo procedimiento, con resultados satisfactorios (Fig. 2).

\section{REFERENCIAS}

1. GRADY RW, CARR MC, MITCHELL ME.: Complete primary closure of bladder exstrophy. Urol Clin North Am 1999; 2695109.

2. GRADY RW, MITCHELL ME.: New concepts in bladder exstrophy and epispadias reconstruction. Dialogues in paediatric urology, 1999; 22 (3).

3. MITCHELL ME, BÄGLI DJ.: Complete penile disassembly for epispadias repair: the Mitchell technique. J Urol 1996; 155: 300-304.

4. HENDREN WH.: Exstrophy of the bladder: An alternative method of management. $J$ Urol 1976; 115: 195.

. KELALIS PD.: Urinary diversion in children by the sigmoid conducit: its advantages and limiation. J Urol 1974; 112: 666.

6. KING LMR, WENDEL EF.: Primary cystectomy and permanent urinary diversion in the treatment of exstrophy of the urinary bladder. In Scott R, Jr., Gordon H.L., Scott F.B., et al (eds): Philadelphia, W.B. Saunders Co., 1972: 244-250.

7. SPENCE HM, HOFFMAN WW, PATE VA.: Exstrophy of the bladder. Long term results in a series of 37 cases treated by ureterosigmoidostomy. J Urol 1975; 114; 133.

8. YOUNG HH.:Exstrophy of the bladder. Surg Gynecol Obstet 1942; 774: 729 .

9. JEFFS RD.: Extsrophy and cloacal exstrophy. Urol Clin North Am 1978; 5: 127.

10. COHEN SJ.: Ureterozystoneostomie eine neve antirefluxtechnik. Aktuelle Urologie 1975; 6: 24.

11. RINKER JR, WITHERINGTON R.: Adaptation of the Lapides principle for incontinence due to anatomical defects. $J$ Urol 1970; 103 (4): 469-9.

12. MARSHALL VF, MARCHETTI AA, KRANTZ KE.: The correction of stress incontinence by simple vesicourethral suspension. Surg Gynecol Obstet 1949; 88: 509.

13. MARTÍNEZ PIÑERIO JA.: Traitement de L'exstrophy vèsicale par entérotrigonourétroplastie. Technique personalle. Acta Urol Bel 1962; 30: 504.

14. MARTINEZ PIÑEIRO JA.: Neovejiga sigmoidea funcionante como tratamiento dse la exstrofia vesical. Arch Esp Urol 1962; 16: 97.

15. LEADBETTER GW. Jr.: Surgical correction of total urinary incontinence. J Urol 1964; 91: 261.

16. ARAP S, GIRON AM, MENEZES DE GOES G.: Initial results of the complete reconstruction of bladder exstrophy. Urol Clin North Am 1980; 7: 477.
17. ALLEN TD, SPENCE HM, SALYER KE.: Reconstruction of the external genitalia in exstrophy of the bladder: preliminary communication. J Urol 1974; 11: 830.

18. JONES JA, MITCHELL ME, RINK RC.: Improved results using a modification of the Young- Dees - Leadbetter bladder neck repair. Br J Urol 1993; 71 (5): 555-561.

19. MOLLARD P.: Bladder reconstruction in exstrophy. $J$ Urol 1980; 124 (4): 525-529.

20. SURER I, BAXER LA, JEFFS RD, GEARHART JP.: Modified Young - Dees - Leadbetter bladder neck reconstruction in patients with succesful primary bladder closure elsewhere: a single institution experience. J Urol 2001; 165 (6 Pt 2): 24382440.

21. DODSON JL, SURER I, BAKER LA, JEFFS RD, GEARHART RD.: The newborn exstrophy bladder inadequate for primary closure: evaluation management and outcome. J Urol 2001; 165 (5): 1656-1659.

22. EL-SHERBINY MT, HAFEZ AT, GHONEIM MA.: Complete repair of exstrophy: Further experience with neonates and children after failed initial closure. J Urol 2002; 168 (4Pt2): 1692-1694.

23. GEARHART JP.: Complete repair of bladder extsrophy in the newborn: complications and management. J Urol 2001; 165 (6 Pt 2): 2431-2433.

24. NICHOLLS G, DUFFY PG.: Anatomical correction of the exstrophy-epsipadias complex: analysis of 34 patients. $\mathrm{Br} \mathrm{J}$ Urol 1998; 82 (6): 865-869.

25. CONNOLLY JA, PEPPAS DS, JEFFS RD, GEARHART JP. Prevalence and repair of inguinal hernias in children with bladder exstrophy. J Urol 1995; 154 (5): 1900-1901.

26. STRINGER MD, DUFFY PG, RANSLEY PG.: Inguinal hernias associated with bladder exstrophy. $\mathrm{Br} J$ Urol 1994; 73 (3): 308-309.

27. HUSMANN DA, MC LORIE GA, CHURCHILL BM, EIN SH.: Inguinal pathology and its association with classical bladder exstrophy. J Pediatr Surg 1990; 25 (3): 332-334.

28. RANSLEY PG, DUFFY PG, WOLLIN M.: Bladder exstrophy closure and epispadias repair. En: Operative SurgeryPaediatrc Surgery, $4^{\text {th }}$ ed. London: Butterwoths 1989: 620.

29. CANTWELL FV.: Operative treatment of epispadias by transplantation of the urethra. Ann Surg 1895; 22: 689.

30. Gearhart JP, Leonard MP, Burgers JH, Jeffs RD.: The Cantwell - Ransley technique for repair of epispadias. $J$ Urol 1992; 148 (3): 851-854.

31. YOUNG HH.: An operation for the cure of incontinence associated with epispadias. J Urol 1922; 7: 1.

32. KRAMER SA, MESROBIAN HGJ, KELALIS PP.: Long term followup of cosmetic apperance and genital function in male epispadias: review of 70 cases. $J$ Urol 1986; 135: 543.

33. MC LORIE, G., PIPPI-SALLE, J. MERGURIAN, P, BAGLI, D, KHOURY A.: Simultanoeus repair of bladder exstrophy and epispadias in male infants. J Urol 1998; 159 (5): 43.

34. GIL VERNET JM.: A new technique for surgical correction of vesicoureteral reflux. J Urol 1984; 131: 456-458.

35. VELASCO B, MARTINEZ URRUTIA MJ, LÓPEZ PEREIRA P, JAUREGUIZAR E.: The effectivenes of the trigonoplasty in the treatment of the primary vesicoureteral reflux. Cir Pediatr 1997; 10 (2): 46-48.

Dr. E. de la Peña

Servicio de Urología Pediátrica.

Fundación Puigvert

Cartagena, 340-350

08025 Barcelona

(Trabajo recibido el 23 abril de 2003) 